\title{
Acoustic investigations of concert halls for rock music
}

\section{Adelman-Larsen, Niels Werner; Thompson, Eric Robert; Gade, Anders Christian}

Published in:

Journal of Acoustical Society of America

Link to article, DOI:

$10.1121 / 1.2942489$

Publication date:

2007

Document Version

Publisher's PDF, also known as Version of record

Link back to DTU Orbit

Citation (APA):

Adelman-Larsen, N. W., Thompson, E. R., \& Gade, A. C. (2007). Acoustic investigations of concert halls for rock music. In Journal of Acoustical Society of America (Vol. 122 (5)). Acoustical Society of America.

https://doi.org/10.1121/1.2942489

\section{General rights}

Copyright and moral rights for the publications made accessible in the public portal are retained by the authors and/or other copyright owners and it is a condition of accessing publications that users recognise and abide by the legal requirements associated with these rights.

- Users may download and print one copy of any publication from the public portal for the purpose of private study or research.

- You may not further distribute the material or use it for any profit-making activity or commercial gain

- You may freely distribute the URL identifying the publication in the public portal

If you believe that this document breaches copyright please contact us providing details, and we will remove access to the work immediately and investigate your claim. 


\title{
Session 1aAA
}

\section{Architectural Acoustics: Sound Systems in Large Rooms and Stadia}

\author{
David S. Woolworth, Cochair \\ Oxford Acoustics Inc., 356 CR 102, Oxford, MS 38655 \\ Timothy W. Leishman, Cochair \\ Brigham Young Univ., Dept. of Physics and Astronomy, Provo, UT 84602
}

\section{Invited Papers}

\begin{abstract}
8:45
1aAA1. Acoustical and sound system design considerations in large venues and stadia. Jack Wrightson, Ron Baker, and Kevin Day (WJHW, Inc., 4801 Spring Valley Rd, \#113, Dallas, TX 75244, rbaker@wjhw.com)

Large, spectator seating facilities provide unique challenges for the sound and acoustical system designer. The challenges are equally daunting, but different for indoor and outdoor facilities. This presentation provides empirical data of existing facilities in regard to speech intelligibility, uniformity of sound reinforcement coverage, maximum loudness, etc., along with a review of predicted modeling and auralization techniques. Design guidelines for acoustical treatment, loudspeaker configuration, predictive calculation of speech intelligibility, and other aspects will be discussed. Difference or variations in design practice between more conventionally sized spaces and very large environments will be presented.
\end{abstract}

\section{9:10}

1aAA2. The implications of scale on sound systems in large spaces. Bob McCarthy (Alignment and Design Inc., 204 Falling Leaves Ct., Creve Coeur, MO, 63141, bob.mccarthy@charter.net)

The symphonic concert hall is a well documented reference point for matters of scale in the musical side of architectural acoustics. Larger spaces may have comparable shapes, and yet the matters of scale require vastly different approaches in regard to the sound system and architectural acoustics. The causes and consequences of scalar expansion will be discussed as well as the shifting balance between the roles of the sound system and the architectural acoustics. Sound system and acoustical designs must adapt to those aspects which are modified by scale (power, distance, wavelength, etc.) along with those that remain constant, regardless of the size (angular aspects, echo perception, the spacing between and size of audience members, etc.). Expanded room scale requires the sound system to use increasingly effective methods of controlling the direct sound field to tailor its response to the highly asymmetric shapes presented for coverage. The methods of control and their implication on the hall acoustics will be discussed. Scalar independent methods for sound system designs which can be form-fitted into a space will be discussed in the context of a variety of large room examples.

\section{9:35}

1aAA3. Stadium acoustics-design challenges and solutions. David Marsh (PMK Consultants, 1420 W. Mockingbird Ln., Ste. 400, Dallas, TX 75247, David.Marsh@pmkconsultants.com)

Large stadiums, whether enclosed or open-air, present many acoustical design challenges including excessive reverberation, echoes from surfaces distant from the sound system loudspeakers, sound absorption by air, refraction, difficulty achieving acceptable speech intelligibility, and synchronization of the sound reinforcement system to the video boards. This paper is an overview of how these challenges have been met in several professional sports facilities. Reverberation times for these large venues are normally underpredicted on the order of $-20 \%$ by conventional methods. Low frequency reverberation times (down to $63 \mathrm{~Hz}$ ) tend to be extremely long - about $15 \mathrm{~s}$ on average. A novel approach will be described to estimate these values (within a wide range of possibilities) based on in situ measurement data. It will be shown that after exhausting all possibilities of acoustical treatments, the most effective way of achieving acceptable speech intelligibility is to use a distributed sound system with no seat being more than about $80 \mathrm{ft}$ from the nearest loudspeaker.

10:00-10:20 Break

10:20

1aAA4. Purpose built sound systems for large rooms. Neil A. Shaw (Menlo Sci. Acoust., Inc., P.O. Box 1610, Topanga, CA 90290-1610, menlo@ieee.org) and John Monitto (Meyer Sound Labs., Inc., Berkeley, CA 94702)

Sound systems for sports facilities have improved over the years with the development of better loudspeaker systems with increased headroom and reduced distortion at high sound levels. Today's loudspeaker systems can produce cleaner sound with less interference between the devices, when installed either temporarily or permanently in these often very reverberant venues. Two basic design philosophies for football, baseball, and soccer stadiums are the point source and the distributed system. Smaller basketball, 
hockey and other sports typically have sound systems flanking scoreboards in a point source design with the option of distributed delayed speakers. In some new facilities that are designed for multipurpose use, the use of electronic processing systems enables these rooms to be designed with lower reverberation times, which is typically the preferred acoustic environment for live amplified concerts. For games and other events a variable acoustic system provides additional reverberation as needed, also enhancing natural crowd and court sounds, such as a "puck" sound, and increases the intimacy of applause and cheering between the fans and the game itself.

\title{
$10: 45$
}

1aAA5. Signal-to-noise ratios in sound system design. Marshall Long (Marshall Long Acoust., 13636 Riverside Dr., Sherman Oaks, CA 91423, mlacoustics@sbcglobal.net)

An important consideration in sound system design is intelligibility, and the most common measure of intelligibility is some sort of signal-to-noise ratio. Intelligibility can be expressed in terms of a goodness ratio (high signal-to-noise) or a badness ratio (high noise-to-signal), or a modified badness ratio (noise $x$ reverberation time)/signal such as \%ALcons. The trick is how to calculate signal, how to calculate noise, and how to design systems with what we calculate. Three types of solutions: Cabinets (horns), distributed loudspeakers, and line arrays are discussed.

\section{1:10}

1aAA6. Live sound measurements in stadia. Wolfgang Ahnert, Stefan Feistel, Alexandra Radu Miron, and Enno Finder (Ahnert Feistel Media Group, Arkonastr. 45-49, D-13189 Berlin, Germany, wahnert@ada-acousticdesign.de)

In this presentation, the authors introduce the software-based measuring system EASERA SysTune to be used for measurements by excitation with music or speech signals. It investigates the use of standard signals supplied from a sound system in a stadium in real-time. Using a newly developed program module, live-sound recordings or speech and music signals from a microphone input and from the mixing console can be utilized to obtain impulse response data for further evaluation. New noise suppression methods are presented that allow these impulse responses to be acquired in full-length, even in occupied venues. As case studies, acoustic measurements based on live sound supply are discussed for a soccer stadium. Required measuring conditions and limitations are derived as a result.

TUESDAY MORNING, 27 NOVEMBER 2007

GRAND COUTEAU, 8:00 TO 10:05 A.M.

Session 1aAO

\section{Acoustical Oceanography: Marine Sediment Properties and Inversions}

\author{
Jon M. Collis, Chair \\ Woods Hole Oceanographic Inst., Bigelow Bldg, Woods Hole, MA 02543
}

Chair's Introduction-8:00

Contributed Papers

8:05

8:20

1aAO1. The effect of grain shape on the porosity of marine sediments. David R. Barclay and Michael J. Buckingham (Marine Physical Lab., Scripps Inst. of Oceanogr., Univ. of California, San Diego, 9500 Gilman Dr., La Jolla, CA 92093-0238)

The porosity of marine sediments increases as the mean grain diameter decreases. In contrast, the close-packing structures (including random packing) of uniform-size spheres all show a porosity that is independent of sphere size. Hamilton suggested that, in sediments, the shape of the grains is instrumental in determining the porosity. To investigate this idea, experiments have been performed on a variety of sand samples (marine sediments, beach sands, and desert sands) with mean radii within the range $30 \mathrm{~mm}-200 \mathrm{~mm}$. The macroscopic properties (porosity and bulk density) are measured in simple tabletop experiments, while the microscopic details of grain shape are quantified using an optical microscope and imageanalysis software. For each grain in, typically, a sample of several hundred, the boundary is traced and various geometric parameters are returned, including the perimeter, the cross-sectional area, the centroid, and the best-fit ellipse. Also, a Fourier decomposition yields the radius as a function of angle, and the higher-order coefficients are then combined to provide a description of grain shape (i.e., deviation from sphericity). An attempt is being made to interpret the observed porosities in terms of the measured grain shapes. [Research supported by ONR.]
1aAO2. Shear waves and the discrepancy between perceived and ideal frequency power laws for sediment attenuation. Jon M. Collis, Allan D. Pierce, and William M. Carey (Boston Univ., Boston, MA 02215, jcollis@bu.edu)

Inverse techniques based on data for long-range propagation in shallow water have recently inferred that the attenuation in certain marine sediments varies at low frequency as the 1.8 power. Idealized models predict the exponent to be exactly 2.0. The inverse inferences usually assume the bottom is a fluid, and this is ordinarily a good approximation, because the shear wave speed in bottom sediments is typically very small. Direct numerical simulation [J. M. Collis et al., Proc. Oceans 2007, Aberdeen (2007)] indicates that shear waves make a sufficient contribution to shallow water attenuation that could account for the small discrepancy in exponents. To better assess whether this is the case, the present paper analyzes the effect of shear waves on modal attenuation. The Pekeris model with a lower elastic half-space is used with the shear wave speed taken to be substantially less than the sound speed in water. The derived dispersion relation has complex roots for the horizontal wave number, and the imaginary part, found by a perturbation analysis, predicts that the shear wave contribution to the modal attenuation is proportional to the cube of the ratio of the shear wave to water sound wave speeds. [Work supported by ONR.] 
1aA03. Complex-density, equivalent-fluid modeling of acoustic interaction with the seafloor. Michael Vera (Univ. of Southern Mississippi, 118 College Dr., \#5046, Hattiesburg, MS 39406, michael.vera@usm.edu)

Acoustic interaction with the seafloor can generate both compressional and elastic shear waves in the solid. Accurate models of shear propagation are often computationally expensive and difficult to apply to long-range propagation. When the sound field in the water is of primary interest, an equivalent-fluid model of the seafloor, with parameters chosen to match the reflection coefficient of the actual elastic solid, can sufficiently characterize the effect of the bottom on energy in the water. The effective density of the seafloor material in this approach can be a complex number. Prior methods for generating equivalent fluids were intended for low shear speeds and low grazing angles. Recent developments in the technique were intended to extend its validity to higher shear speeds and a wider range of angles. These efforts were initially motivated by the need to simulate bottom-interacting arrivals for the broadband Kauai source in the North Pacific Acoustic Laboratory experiment at megameter ranges. The work to be presented involves a more detailed examination of the performance of the method, including comparisons to benchmark models and to shorter range data from this Kauai source collected as part of the Basin Acoustic Seamount Scattering Experiment.

\section{8:50}

1aAO4. A direct inversion scheme to obtain sediment sound speed and density in shallow water. Kyle M. Becker (Penn State Univ./Appl. Res. Lab., P.O. Box 30, State College, PA 16804-0030)

A simple inversion scheme is described for estimating the sound speed and density in the seabed for Pekeris-type waveguides. At a fixed frequency, input data to the inversion algorithm are discrete values of the phase of the plane wave reflection coefficient at the water/bottom interface. Above the critical angle, the input data are derived from estimates of the horizontal wave numbers for propagating modes in the wave guide. For two or more propagating modes, a linear system of equations is solved directly to determine sound speed and density in the bottom. The approach is strictly only valid for determining properties of the lower half-space in a Pekeris Wave guide. However, in certain circumstances, the approach can be applied to determine properties of the topmost sediment in layered media. The method is demonstrated on a synthetic data set and then applied to data collected at sea. [Work supported by an ONR Ocean Acoustics Entry-Level Faculty Award.]

\section{9:05}

1aA05. Analysis of the effect of water column sound speed variation on geoacoustic inversion. Yong-Min Jiang and N. Ross Chapman (School of Earth and Ocean Sci., Univ. of Victoria, PO Box 3055, Victoria, BC V8W 3P6, Canada)

Bayesian matched field inversion has been applied on multi-tonal data sets acquired on the New Jersey continental shelf in the SW06 experiment in August, 2006. Since the data sets were collected in a range-dependent environment due to water column sound speed variation, sound speed profile (SSP) was decomposed in terms of empirical orthogonal functions and also inverted in the inversion. This presentation examines the sensitivity of the geoacoustic and geometrical parameters to the shape and the gradient of the sound speed in the thermocline by the interparameter correlation from the inversion. The effects of the SSP on the geoacoustic inversion and source localization are studied through the inversions of measured data at different ranges with/without prior information about geometric parameters in the inversion. Further investigations on the variation of SSP to the sediment sound speed, density, and attenuation at different ranges are carried out by simulations. It is found that the geometric parameters are more sensitive to the SSP than are the geoacoustic parameters in this shallow water environment. [Work supported by ONR.]
1aAO6. Geoacoustic information content of bottom-moored horizontal line array data. Dag Tollefsen (Norwegian Defence Res. Establishment (FFI), Box 115, NO3191 Horten, Norway) and Stan E. Dosso (School of Earth and Ocean Sci., Univ. of Victoria, Victoria BC, Canada V8W 3P6)

This paper considers geoacoustic information content in matched-field inversion of acoustic data from bottom-moored horizontal line arrays. Bayesian geoacoustic inversion is applied to low-frequency multi-tone data in a synthetic shallow water environment under realistic signal-tonoise ratios and for frequency bands relevant to both controlled-source experiments and ship-noise inversion. The information content is quantified in terms of widths of marginal a posteriori probability densities of model parameters obtained by fast Gibbs sampling. The information content dependence on factors such as array length and sensor configuration, source range and bearing (from endfire to broadside), and frequency content is studied.

\section{9:35}

1aA07. Bayesian geoacoustic inversion of ship-noise data from a bottom-moored horizontal line array. Dag Tollefsen (Norwegian Defence Res. Establishment (FFI), Box 115, NO3191 Horten, Norway) and Stan E. Dosso (School of Earth and Ocean Sci., Univ. of Victoria, Victoria BC, Canada V8W 3P6)

This paper presents results from matched-field geoacoustic inversion of low-frequency $(40-150 \mathrm{~Hz})$ ship-noise data recorded on a horizontal array deployed at the seafloor. Data are taken from an experiment conducted by FFI in shallow waters of the Barents Sea. Estimates of sediment parameters were obtained by inversions of narrowband data from a relatively quiet surface ship at source-array ranges of 1-6 km. The fast Gibbs sampler was used to estimate posterior probability densities of model parameters. Estimates of sound speed and density of quaternary sediment are compared with results from inversion of data from a towed source collected along the same track, and with reference values from other geophysical data collected in the area.

\section{9:50}

1aAO8. Improved perturbative inversion schemes for obtaining bottom geoacoustic properties in shallow water. Megan S. Ballard and Kyle M. Becker (Penn State Univ., State College, PA 16802, msd200@psu.edu)

Perturbative schemes using modal wave numbers are used to obtain sound speed in the sediment as a function of depth [S. D. Rajan, et. al., J. Acoust. Soc. Am. 82(3), 998-1017 (1987)]. The inversion algorithm involves solving an ill-posed problem, with regularization and singularvalue decomposition used to stabilize the solution, resulting in a smoothed version of the true sound speed profile. However, owing to geological processes, sediments are often better described by layers having distinct properties and are not well represented by a smooth profile. In this work, two new methods are applied to stabilize the solution of the inverse problem, making it possible to resolve discontinuities in the sound speed profile of the sediment. Piecewise polynomial truncated singular value decomposition (PP-TSVD) is used in place of traditional stabilizing methods and is demonstrated to yield better results when a layered model is a reasonable assumption. The results can be further improved using qualitative regularization if prior knowledge of the location of sound speed discontinuities is available. These methods are shown to yield very accurate estimates of the sound speed profile deep into the sediment using very few perturbations to the forward model. [Work supported by NDSEG and ONR.] 


\title{
Session 1aUW
}

\section{Underwater Acoustics: Propagation Modeling}

\author{
Josette Paquin Fabre, Chair \\ Acoustics Division, Naval Research Lab., Stennis Space Center, MS 39529
}

Chair's Introduction-10:25

Contributed Papers

10:30

1aUW1. Integrated signal excess as a metric for environmental acoustic assessment. J. Paquin Fabre (Naval Res. Lab., 1005 Balch Blvd., Stennis Space Ctr., MS 39529)

Signal excess (SE) is often used as a metric for determining acoustic performance through a waveguide or over an area. This quantity can limit the capability to assess the environment because SE is computed given a single acoustic source, receiver and frequency, and is a function of range from the source, thereby limiting the scenarios for which the environment can be assessed as well as the ability to visualize it over an area. Integrated SE (ISE) with phase tracking is proposed as an improved metric for evaluation of acoustic performance. For a given source location (in latitude and longitude), the SE is integrated over all possible source depths, a band of frequencies, and bands of ranges. Additionally, the phase variations across the regions are tracked. This metric is compared to various traditional SE fields and is shown to provide a better representation of the overall acoustic properties of a waveguide and an area. The ISE and the original SE are also examined across an ensemble of sound speeds and the variations of the acoustic environment are characterized. [The author appreciates and acknowledges the funding support from the Naval Research Laboratory Base Program.]

\section{0:45}

1aUW2. Modeling surface duct precursors for Littoral Acoustic Demonstration Center 2003 (LADC03) seismic calibration experiment. Arslan M. Tashmukhambetov, George E. Ioup, Juliette W. Ioup (Dept. of Phys., Univ. of New Orleans, 2000 Lakeshore Dr., New Orleans, LA 70148, atashmuk@uno.edu), and Natalia A. Sidorovskaia (UL Lafayette, Lafayette, LA 70504-4210)

In summer 2003, LADC conducted a seismic calibration experiment for a 3190-in3 21-element seismic exploration array. After calibration, absolute broadband (up to $25 \mathrm{kHz}$ ) pressure-time dependencies for a wide range of offsets and arrival angles were produced. A computational workflow, combining the upgraded version of the Navy standard acoustic propagation model RAM and industry airgun modeling packages, was developed to model the calibrated experimental data. Experimental and modeled data have demonstrated good agreement in absolute pressure amplitudes and large-scale frequency interference pattern for the frequencies up to $1,000 \mathrm{~Hz}$. The results of the application of the developed workflow for investigation of the effect of a seasonally-formed surface duct on seismic array energy distribution is presented. The presence of the surface duct is experimentally confirmed to be responsible for the generation of a unique set of modes forming the precursor arrivals, first systematically observed from a point source on a near-bottom hydrophone by DeFerrari and Monjo. The influence of a range-dependent environment on precursor stability is addressed. An attempt to identify precursors in the LADC03 experimental data is discussed. [Research sponsored by the Industry Research Funding Coalition and the Joint Industry Project.]
1aUW3. Efficient modeling of range-dependent seismo-acoustics problems. Michael D. Collins, Woo-Yeol Jung (Naval Res. Lab., Washington, DC 20375), Elizabeth T. Kusel (Northeastern Univ., Boston, MA 02115), and William L. Siegmann (Rensselaer Polytechnic Inst., Troy, NY 12180)

The parabolic equation method is the most effective approach for solving range-dependent problems in ocean acoustics. During the past three decades, many difficulties have been encountered while attempting to generalize this approach to problems involving elastic layers. With the development of an improved single-scattering solution [J. Acoust. Soc. Am. 121, 808813 (2007)], a large class of range-dependent seismo-acoustics problems can now be solved accurately and efficiently. Previous singlescattering solutions for seismic problems were based on an iteration formula, and limited by convergence problems. Since the improved solution does not require iteration, it provides the efficiency of the energyconserving solution that has proved to be very useful for acoustics problems. The application of this approach to problems involving fluid layers is currently being investigated. [Work supported by ONR.]

\section{1:15}

1aUW4. Mode formulas for shallow water waveguides using a modified asymptotic approximation. Stephen V. Kaczkowski, William L. Siegmann (Dept. of Mathematical Sci., Rensselaer Polytechnic Inst., 110 8th St., Troy, NY 12180-3590, kaczks@ rpi.edu), Allan D. Pierce, and William M. Carey (Boston Univ., Boston, MA 02215)

Sound speed profiles in shallow water waveguides are small deviations from isospeed and often decrease monotonically with depth. Approximation formulas for the propagating modes are found using a modified form of the classical WKBJ method. The ocean bottom is taken as homogeneous. The approach is accurate for modes with phase speeds greater than, and even slightly less than, the maximum water sound speed. The validity and accuracy of the approximations over frequency and mode number are illustrated using benchmark numerical calculations. Comparisons with approximations from other methods, including previous WKBJ approaches, are described. The formulas here are typically more compact and convenient. The results demonstrate how changes in parameters, such as frequency, bottom sound speed, and water sound speed profile quantitatively affect the mode functions. Applications for representative waveguide profiles are provided. [Work supported by the Office of Naval Research.]

\section{$11: 30$}

1aUW5. Modeling underwater sound propagation from an airgun array using the parabolic equation method. Alexander MacGillivray (JASCO Res. Ltd., 4464 Markham St., Victoria, BC, Canada)

A technique for modeling sound propagation from an airgun array using the parabolic equation (PE) method is presented that takes into full account the far-field, angle-dependent directionality pattern of the array. This is achieved by generating a PE starting field for the array by summing together shaded, phase-shifted replicas of the PE self-starter. The 
array starter has been implemented using the RAM parabolic equation code. Validation comparisons are presented of field predictions generated using the array starter against exact normal mode solutions for an array source computed using ORCA. Examples of synthetic waveform airgun array calculations are also presented. The array starter method can be used to accurately predict pressure waveforms from an airgun array in the ocean environment provided that the modeler knows (or can compute) "notional" far-field source signatures for individual airguns in the array.

\section{1:45}

1aUW6. Model for energy consumption by a broadband shallowwater acoustic communications network. Daniel Rouseff and Warren L. J. Fox (Appl. Phys. Lab., College of Ocean and Fishery Sci., Univ. of Washington, Seattle, WA 98105, rouseff@apl.washington.edu)

A multistatic active sonar system with several widely distributed sensors must share information between the sensors. Acoustic communication is a viable method to share information and is particularly appropriate when the sensors are mobile. A potential drawback is that a significant fraction of the total energy budget for the system might be consumed just by sending information between the sensors. In the present work, an energy consumption model is developed that is appropriate for incoherent acoustic communication in shallow water. The model builds upon the foundation established by Sozer et al. [IEEE J. Oceanic Eng. 25, 72-83 (2000)], but uses an improved sub-model for acoustic propagation. The sub-model includes environmental factors like the sound speed profile in the water column and the composition of the seabed. By exploiting the waveguide invariant concept, the broadband nature of the communications signals can be included efficiently in the calculation. Numerical results demonstrate how relaying messages between intermediate sensors can save substantial energy compared to direct communications. The calculations also show that energy consumption can vary by more than an order of magnitude depending on the seabed composition. [Work supported by ONR.]

\section{2:00}

1aUW7. Relating $\mathrm{X}$-waves and Cerenkov radiation. Shane C. Walker (Univ. of California, San Diego, Scripps Inst. of Oceanog., Marine Phys. Lab., 9500 Gilman Dr., Mail code 0238 La Jolla, CA 92093-0238)

$\mathrm{X}$-waves are shown to be related to Cerenkov-Vavilov (CV) radiation as derived by solving the linearized inhomogeneous scalar wave equation in free space for the case of a source in uniform motion at velocity $\mathbf{v}$ that is greater than the speed of waves, $\mathrm{c}$, in the medium, $|\mathrm{v}|>\mathrm{c}$. The results apply to wave phenomena in general, both acoustic and electromagnetic. In this context, the supersonic/superluminal properties of $\mathrm{X}$-waves are unambiguously demonstrated to be phase effects. One implication is that even the ideal diffractionless $\mathrm{X}$-wave is not signal-like nor can it exceed the Shannon data rate bandwidth relation, $\Delta \mathrm{t} \Delta \omega=2 \pi$. Further, it is shown that the continuous power required to propagate an $\mathrm{X}$-wave is analogous to the energy dissipation of a CV particle caused by radiation drag. Thus, generating a diffractionless $\mathrm{X}$-wave requires an infinite total energy input over a spatially infinite source aperture. Finally, the connection between $\mathrm{CV}$ radiation and $\mathrm{X}$-waves suggests the possibility of generating $\mathrm{X}$-waves using time-reversal.

\title{
Session 1pAA
}

\section{Architectural Acoustics, Musical Acoustics, Signal Processing in Acoustics, and Noise: Even Better Than the Real Thing—Rock, Pop, and All That Jazz}

\author{
Alexander U. Case, Cochair \\ Fermata Audio and Acoustics, P.O. Box 1161, Portsmouth, NH 03802-1161 \\ K. Anthony Hoover, Cochair \\ Cavanaugh Tocci Associates, Inc., 327F Boston Post Rd., Sudbury, MA 01776
}

Chair's Introduction-1:00

Invited Papers

1:05

1pAA1. Psychoacoustic evaluation of music reproduction in passenger cars. Hugo Fastl, Martin Ammler (AG Technische Akustik, MMK, TU Muenchen, Arcisstr. 21, 80333 Muenchen, Germany, fastl@mmk.ei.tum.de), and Adam Sulowski (Audi AG, Ingolstadt, Germany)

A method to assess the quality of music reproduction in passenger cars is developed and evaluated. In essence, the subject sits in a standing car and is presented from a CD music of different genres via the car's audio system. The subject has to rate different aspects of the performance on a 10 step scale using a PDA. From the ratings, a net like a spider net, with different attributes like natural, squeeking, dull, reverberant, acceptable, good etc. on its rays is constructed. The goal is a net with a large area, i.e. as many as possible of the attributes should attain the maximum rating. Examples from a pilot study are given for car audio systems of different sophistication, and aspects like gender differences or possible biases towards a product will be discussed. 
1pAA2. Changing the paradigm of practice rooms and music teachers coaching studios. Ron Freiheit (Wenger Corp., 555 Park Dr., Owatonna, MN 55060)

For years the image of practice rooms for many musicians meant playing in places that at times resembled (and sometimes were, in fact) closets. They served the function by creating individual places to practice, but were usually less than desirable due to the internal acoustics provided. The same paradigm has held true for most music teachers coaching studios. Time spent with the teacher was invaluable for improving one's craft, however the acoustics were constrained the rooms physical dimensions, construction materials and room furnishings. With the advent of DSP, these spaces can now provide a variety of acoustic environments for practice and coaching. Integrated into the latest DSP technology is the ability to provide concurrent recording capability, enabling immediate review of the musician's performance. In the coaching studio, it is especially effective when the student can quickly hear a specific point the teacher is making. New technology accomplishes this more quickly than with past recording methods. The net result of these additional tools is to accelerate the potential learning experience for the musician.

\section{$1: 45$}

1pAA3. Fictional timbre-Close microphones and warped perspectives. Alexander U. Case (Sound Recording Technol., Univ. of Massachusetts, Lowell, 35 Wilder St., Lowell, MA 01854)

Recording engineers, particularly in pop and rock genres, have a long tradition of placing microphones exceptionally close to the sound sources they wish to record. Not a gesture towards realism, close microphone techniques are born of practical needs for sound isolation in sometimes cramped recording spaces, production pressures to defer signal processing decisions until the mixdown session, and creative desires to variously avoid, enhance, and/or exaggerate sonic features of the instrument in search of a sound that is "better than the real thing."

\section{2:05}

1pAA4. Psychoacoustics of non-real spaces. Sam Ortallono (MediaTech Inst., 3324 Walnut Bend Ln., Houston, TX 77042)

Psychoacoustics of non-real spaces, modern recording, and mixing techniques provide the opportunity to create acoustic phenomena that do not exist in the real world. This presentation will discuss how recorded music has been manipulated and try to understand what people think they hear and why. Through a combination of recent original recordings and classic records, investigations can be made into how processed sound can disorient, surprise, or please the listener. In the studio, compressors, gates, delays, and artificial reverbs are employed to create unnatural soundscapes, yet the mind still applies the rules that have been learned in everyday life. Such impossibilities as backward sounds and multiple sources will also be considered. Finally, some speculation can be made as to why these records seem to sound even better than the real thing.

1pAA5. What to do with a five-track woodwind quintet. James W. Beauchamp and Mert Bay (School of Music and Dept. of Elect. and Comput. Eng., Univ. of Illinois at Urbana-Champaign, Urbana, IL 61801, jwbeauch@uiuc.edu)

Recently we made a five-track recording of a woodwind quintet using a two-step process: First, the group was recorded together in the same room with each instrument close-miked. Second, each instrument was recorded separately with the performer monitoring the others on headphones. There were a few synchronization problems, but basically the method worked fine, and the result is a recording with complete separation between the tracks. Although this was inspired by the necessity for ground truth for a demixing project, several other advantages of this approach to recording have come to mind: (1) Listening to all instruments played back simultaneously from separate speakers is very enjoyable. (2) Music-minus-one recordings are useful for musicians to practice with and are easy to produce. (3) Data reduction on each track enables analysis, modification, and resynthesis projects of various sorts. For example, pitch, tempo, relative loudness, and timbre are easy to manipulate. Examples of music produced by processing the woodwind quintet will be demonstrated.

1pAA6. Making outdoor acoustic venues "better than the real thing." Pamela Harght (School of Architecture and Urban Planning, Univ. of Kansas, 1465 Jayhawk Blvd., Lawrence, KS 66045)

Traditionally-acoustic music organizations, particularly orchestras, are developing new ways to reach out to a wider audience, extend their season, and promote their music in non-traditional summer homes. Many of these outdoor venues accommodate far more patrons than the established, enclosed concert hall. Furthermore, with these larger venues comes the responsibility to enhance the performing group through amplification to satisfy the expectations of the patrons and to compensate for the vastly different atmosphere regular concert-goers are accustomed to. Electronic reinforcement is now required to accommodate the thousands more outdoors and with digital reverberation and modern recording equipment, these goals and expectations can be met with little compromise in the listening experience. This paper will discuss the role of sound reinforcement in orchestral music, focusing on the popular Tanglewood Music Center, the summer home of The Boston Symphony Orchestra, and other successful venues. 
1pAA7. Pulse and swing: Quantitative analysis of hierarchical structure in swing rhythm. Ken Lindsay (tlafx, 180 Ohio St., Ashland, OR 97520, samba4ken@gmail.com) and Pete Nordquist (Southern Oregon Univ., Ashland, OR 97520)

Swing feeling in music is generally attributed to the presence of timing variations in the rhythm as played, contrasted with standard tablature form. Substitution of triplet subdivision for the divide by two format (quarter, eighth, sixteenth notes) of MozartBach (MB) notation is a well known timing variation associated with Swing. We present evidence that an important part of Swing is also found in nonuniform timing of the Pulse, or basic beat, of a Swing tune i.e., time delta between downbeats of successive musical measures does not conform to a regularly spaced time grid as defined by the metronome. To our knowledge, this fundamental aspect of Swing has not been previously reported. The departure from a uniform time grid and presence of different types of time variations played by different instruments both make important contributions to the "lively" quality found in Swing style of many genres: Classic Jazz Swing, Pop, Gospel/Soul, Rock and Roll, Samba, etc. Although different rhythms may not be locked together as in MB style, nonetheless they are all highly synchronized. The degree of synchronization corresponds to varying degrees of tight or loose rhythmic style. This also contributes to the "live" feeling in the musical performance.

$3: 35$

1pAA8. Reality is not a recording/a recording is not reality. Jim Anderson (New York Univ., 194 Mercer St., Rm. 501, New York, NY 10012)

The former New York Times film critic, Vincent Canby, wrote all of us have different thresholds at which we suspend disbelief, and then gladly follow fictions to conclusions that we find logical. Any recording is a fiction, a falsity, even in its most pure form. It is the responsibility, if not the duty, of the recording engineer, and producer, to create a universe so compelling and transparent that the listener isnt aware of any manipulation. Using basic recording techniques, and standard manipulation of audio, a recording is made, giving the listener an experience that is not merely logical but better than reality. How does this occur? What techniques can be applied? How does an engineer create a convincing loudspeaker illusion that a listener will perceive as a plausible reality? Recordings will be played.

3:55

1pAA9. Audio networks-From the main stage to main street. Deb Britton (K2 Audio, LLC 4900 Pearl East Cir., Ste. 201E, Boulder, CO 80301)

In recent years, digital audio networks have become a more and more prevalent means of distributing audio signals. Audio networks are not unique to large, permanently-installed sound systems, but are also used in touring sound, homes, and even some musical instruments. The audio network trend was not sparked by a desire to be on the cutting edge of technology, but to fill some very real needs. Audio technology has been steadily moving from analog to digital, yet the interconnection of the individual components remains in the analog domain. The resultant numerous $\mathrm{A} / \mathrm{D}$ and $\mathrm{D} / \mathrm{A}$ conversions can cause signal quality issues, severely limiting the performance of the system. A network provides a common, digital interconnection, and reduces the infrastructure requirements by routing many signals over fewer cables. For the facility owner, the greatest benefit could be the drastic reduction in cabling. For the engineer or system end user, any input to any output routing capability enables the implementation of different system configurations on the fly, and can allow for future expansion, without the need for hardware or wiring changes. For the musician or home studio enthusiast, a networked instrument provides a direct interface to a computer for recording.

4:15

1pAA10. A survey of small live music venues of New Orleans. David Woolworth (Oxford Acoust., Inc., 356 CR 102, Oxford, MS 38655, dave@oxfordacoustics.com)

This survey of small live music venues of New Orleans will summarize aspects of history, capacity, sound reinforcement specifications, and acoustic properties. There will also be information on the factors affecting sound quality for small venues and some examples of "on the fly" problem solving, using available materials and sound processing.

\section{Contributed Papers}

4:35

1pAA11. Acoustic investigations of concert halls for rock music. Niels W. Adelman-Larsen (Flex Acoust., Scion-DTU, Diplomvej 377, 2800 Lyngby, Denmark, nwl@flexac.com), Eric R. Thompson, and Anders C. Gade (Tech. Univ. of Denmark, 2800 Lyngby, Denmark)

Objective measurement data and subjective evaluations have been collected from 20 small-/medium-sized halls in Denmark used for amplified rhythmic music concerts (pop, rock, jazz). The purpose of the study was to obtain knowledge about optimum acoustic conditions for this type of hall. The study is motivated by the fact that most concert tickets sold in Denmark relate to concerts within these genres in this kind of venue. The subjective evaluations were carried out by professional musicians and sound engineers who responded on the basis of their experiences working in these (and other) halls. From the relationships between the subjective evaluations and the objective data, recommended values for reverberation time across frequency are suggested. For halls with volume between $50.000 \mathrm{ft}^{3}$ and $160.000 \mathrm{ft}^{3}$, the optimal range is $0.7 \mathrm{~s}$ to $1.0 \mathrm{~s}$ without audience (and chairs). T30 should be frequency independent, so that clarity remains high also at the lower frequencies. A lower limit for T30 was identified. The paper also proposes possible hall designs that seek to fulfill the demands of sound engineers and musicians, as found in the investigation. 
paper presents field performance test results for several small businesses that failed to meet the regulatory noise limits and were in jeopardy of being fined or closed down until they could comply with the regulatory noise requirements. The businesses all provided musical entertainment, and were located on busy streets in commercial buildings. All of the businesses also had a customer base that expected loud music. Most of the businesses were afraid that reducing the volume of their musical entertainment would cause them to lose customers and money. Several solutions were explored and recommendations made that satisfied both sides of the issue.

TUESDAY AFTERNOON, 27 NOVEMBER 2007

NAPOLEON C3, 1:55 TO 4:30 P.M.

\title{
Session 1pAB
}

\section{Animal Bioacoustics: Acoustics of Bats, Birds, Insects, and Rodents (Lecture/Poster Session)}

\author{
Mardi C. Hastings, Chair \\ Pennsylvania State Univ., Applied Research Lab., State College, PA 16804
}

Chair's Introduction-1:55

\section{Contributed Papers}

2:00

1pAB1. Link-based feature extraction for model-based detection of echolocation calls. Mark D. Skowronski and M. Brock Fenton (Biol., Univ. of Western Ontario, London ON N6A 5B7, Canada, mskowro2@uwo.ca)

Frame-based acoustical analysis of echolocation calls from bats has recently been proposed to improve the robustness of automated call detection. Statistical models, popularized and honed by automated human speech processing research, learn the distributions of call features extracted identically from short overlapping frames of time. A fundamental assumption is that each frame contains at most one echolocation call. In field recordings of bats, this assumption is often violated by the presence of echoes and overlapping calls from multiple bats, which degrade automated detection performance. Simple rules, based on expert knowledge of echolocation calls, were used to follow local spectrogram ridges and to group ridge points into links. Frame-based features were extracted from the links, which may overlap in time, allowing links to be used in the existing model-based analysis paradigm. Improved performance using links is demonstrated for several pathological cases, including conspecific interaction and high-duty-cycle foraging.

\section{2:15}

1pAB2. Simulating bat echolocation. Fons De Mey, Jonas Reijniers, and Herbert Peremans (Universiteit Antwerpen, Labotheek, Prinsstraat 13, 2000 Antwerpen, Belgium, fons.demey@ua.ac.be)

In accordance with the growing practice of performing acoustical experiments in virtual reality, the acoustic properties of the echolocation system of a number of bat species are characterized in simulation. The head related transfer function (HRTF) and interaural intensity differences (IIDs) from the pinnae and the radiation pattern from the noseleaves are calculated with a boundary-element method. The obtained results are compared to actual acoustic measurements performed on other specimens of the same species. This comparison shows that performing the experiments in virtual reality is a viable alternative to performing measurements on real specimens. It has the additional advantage that the spatial resolution that can be achieved compared to the more commonly used acoustical measurements, is higher. Furthermore, computer manipulation of the virtual morphology model allows characterizing the influence of different head parts on the HRTF and the noseleaf radiation pattern. To illustrate the power of this technique, the effects of different head parts on the HRTF of the phyllostomid bat Phyllostomus discolor are investigated. For this species, it is concluded that the pinna has a larger effect on the interaural intensity difference (IID) pattern than the head itself.

\section{2:30}

1pAB3. Frequency-swept directivity lobes-An emerging functional principle of biosonar beamforming. Rolf Müller, Hongwang Lu, Zhiwei Zhang (School of Phys. \& Microelectronics, Shandong Univ., Hongjia Lou 5, 250100 Jinan, China, mueller@sdu.edu.cn), and John R. Buck (Univ. of Massachusetts Dartmouth, North Dartmouth, MA 02747)

In all bat species, the spatial sensitivity of the biosonar system is mainly determined by the shape of the outer ears. In addition, a noseleaf surrounds the nostrils in many species and shapes the spatial distribution of the emitted sound energy. Predicting the beam-forming effects of these structures numerically from their surface geometry has enabled comparative studies of high-resolution beam pattern estimates across a large set of different bat species. The beam patterns obtained so far differ from common manmade transducers, such as loudspeakers and microphones. Strong asymmetric side lobes, which change direction as a function of frequency, are a distinguishing feature consistently seen in emission and reception beam patterns of the bats. Where investigated, the occurrence of these side lobes appears to be tied to the presence of flap-like structures (including the tragus of the pinna), which may be hypothesized to have evolved for this purpose. The presence of these side lobes suggests that bat biosonar has not been evolved to maximize the ensonification energy and sensitivity for a single small target unconditionally. Instead, the function of these strong side lobes could be explained as maximizing the information obtained in analogy to squinted beams used in manmade systems. 
1pAB4. Sound recordings from the 2007 emergence of Brood XIII cicada. Ralph T. Muehleisen (Civil, Architectural, and Environ. Engr., Illinois Inst. of Technol., 3201 S. Dearborn St., Rm. 228, Chicago, IL 60616, muehleisen@iit.edu)

Level calibrated recordings of the Brood XIII 17-year periodic cicadas were made in June, 2007 in River Forest, Illinois. Brood XIII consists of three separate species: Magicicada cassini, M. septendecim, and the more rare $M$. septendecula. Power spectrum analysis of a $2 \mathrm{~min}$ segment of the recording shows a $5.95 \mathrm{kHz}$ resonance with $Q \approx 6$, indicative of cassini, and a $5 \mathrm{~dB}$ lower resonance at $1.35 \mathrm{kHz}$ with a $Q \approx 13.5$, indicative of septendecim. Since other researchers have estimated that the power of septendecim is $20 \mathrm{~dB}$ below that of cassini, the high level of the septendecim song suggests that 30 times more septendecim than cassini were singing. Computation of the sound pressure level shows a $2-5 \mathrm{~dB}$ variation with a period of $7.5 \mathrm{~s}$, indicative of synchronized chorus singing of cassini.

\section{3:00-3:15 Break}

\section{3:15}

1pAB5. Possible vocalizations of the ivory-billed woodpecker (Campephilus principalis). Michael D. Collins (Dept. of Mathematical Sci., Rensselaer Polytechnic Inst., Troy, New York 12180), Richard S. Martin (529 Durham Dr., Homewood, AL 35209), and Dalcio K. Dacol (19149 Hempstone Ave., Poolesville, Maryland 20837)

The Ivory-billed Woodpecker (Campephilus principalis) was feared to be extinct for decades, but there have been sightings in several states during the past few years. During a study at the last known nest sites in the 1930s, the characteristic kent calls of this species were recorded and other calls were observed but not recorded. There have been recent observations and recordings of high-pitched calls that are suspected to be ivorybill vocalizations. In the Pearl River, the calls were heard mixed in with kents during an encounter with two ivorybills. At the same site two days later, the calls were heard and recorded after an ivorybill was flushed, and the movements of the source of the calls was correlated with the movements of the ivorybill (video was also obtained during the encounter). Similar calls were recorded along with kent-like calls in the Choctawhatchee River at a site where there were sightings the same day and the previous day. The calls were also heard in the Congaree Swamp after a recording of kents was played back. The calls are similar to one of the calls of the Blue Jay (Cyanocitta cristata), but this conspicuous species was not observed during the encounters.
1pAB6. Design of LabVIEW-based software for insect acoustic research. Vijay Ramalingam, Tom Fink, John Seiner (The Natl. Ctr. for Physical Acoust., 1 Coliseum Dr., University, MS 38677, virjay@olemiss.edu), Douglas Streett (ARS-USDA, Stoneville, MS), and Alan Lax (ARS-USDA, New Orleans, LA)

LabVIEW-based software has been developed for acquiring and analyzing acoustical data from insects under field and laboratory conditions. The following features will be discussed in the presentation: Data acquisition using single or multiple transducers using National Instruments hardware. Timed, threshold, and periodic data acquisition routines. Periodical playback of WAV files or sine waves during data acquisition. Embedded META data information to avoid field notebooks. Real-time saving or deleting acquired data without losing acquisition continuity. Sequential, logical, and automatic file saving routines with time and date stamped file names. Playback of acquired data at different speeds $(0 \leqslant v \leqslant 1)$, and at different time frames. Compare multiple waveforms (up to four) using synchronized pan and zoom routines. Create annotations, image file, and small segments of the original waveform with a single mouse click. Create spectrograms, FFT, and power spectrums of the acquired data. [Work supported by USDA ARS.]

\section{$3: 45$}

1pAB7. A vowel identification procedure for gerbils. Joan M. Sinnott and Kelly W. Mosteller (Psych. Dept., Univ. of South Alabama, Mobile, AL 36688)

Mongolian gerbils are small rodents with auditory specializations that provide them with excellent hearing in the human speech range. Our lab has previously developed simple go/no-go response procedures to measure gerbil detection and discrimination of human vowels. Here is presented our new identification procedure that uses a two-alternative, forced-choice, left versus right response. The gerbil is tested in a small wire mesh cage that contains a center platform, a light, and two feeder cups left and right of the light. The gerbil begins a trial by mounting the platform when the light flashes. One of two vowel sounds (e.g. /u/ or /i/) then starts repeating at the rate of 1 per sec. The gerbil is reinforced with food if s/he goes to the left feeder cup for $/ \mathrm{u} /$, or to the right feeder cup for /i/. Incorrect responses are punished with timeouts. Gerbils easily learn to differentiate the spectrally-dissimilar vowels /u/ versus /i/, and data will be presented on their ability to differentiate more similar vowels such as /a/ versus /ae/. Gerbil identification ability appears related primarily to second formant frequency differences. A video will be shown of gerbils working on the procedure. [Research supported by NIH.]

All posters will be on display and all authors will be at their posters from 4:00 p.m. to 4:30 p.m.

1pAB8. The substrate vibration generating behavior of Aphaenogaster carolinensis (Hymenoptera: Formicidae). Timothy O. Menzel (Natl. Ctr. for Physical Acoust., Univ. of Mississippi, University, MS 38677), Jake R. Marquess (Univ. of Mississippi, University, MS 38677), John Seiner (Natl. Ctr. for Physical Acoust., Univ. of Mississippi, University, MS 38677), and Douglas Streett (USDA-ARS-BCMMUR, Stoneville, MS)

Aphaenogaster carolinensis Wheeler produces substrate vibrations by striking a substrate with its mandible and dragging its mandible across the surface. We have described this behavior and conducted laboratory trials to determine the conditions under which it occurs. Individual strikes were consistent in pattern and duration, but there was great variation in the duration of, and the number of strikes associated with, a single substrate vibration generating event. In laboratory trials, this behavior occurred most frequently in response to confrontation with non-nest mate conspecifics and did not require the presence of a food source or territory to be initiated.
1pAB9. Buzz digging and buzz plastering in the black-and-yellow mud dauber wasp, Sceliphron caementarium (Drury). Tom Fink, Vijay Ramalingam, John Seiner (NCPA, Univ. of Mississippi, One Coliseum Dr., University, MS 38677, tfink@olemiss.edu), Niels Skals (Univ. of Southern Denmark, DK-5230 Odense M, Denmark), and Douglas Streett (USDA-ARS, Stoneville, MS 38776)

The black-and-yellow mud dauber digs mud with its mandibles at pond or puddle edges, flies with a mud ball to a locality that is dark and protected from rain, and then immediately begins to form a mud tube for eventual provisioning with paralyzed spiders as food for its single developing larva (only one egg is depositied per tube). Digging and plastering (making a mud tube) are always accompanied by intense vibrations (intense enough to shake one's hand when holding the wasp with forceps) caused by the wing muscles. Visual and acoustic details of digging and plastering were studied by high speed video (usually 2,000 fps) correlated with acoustical data recorded with laser Doppler vibrometry, microphones, and accelerometers. High definition video was also used to view the general process of digging and plastering, but it is impossible to visualize the 
vibrations except with high-speed video. The vibration of the wing muscles causes the head to vibrate at the same frequency, which mechanically aids digging and plastering. Pulling and pushing mud with the man- dibles while digging are at different frequencies, and plastering is at an even lower frequency. The wasp can modulate the amplitude of these vibrations while digging or plastering.

TUESDAY AFTERNOON, 27 NOVEMBER 2007

GRAND COUTEAU, 1:35 TO 3:45 P.M.

\title{
Session 1pAO
}

\section{Acoustical Oceanography: Storms and Intense Air-Sea Interactions}

\author{
Jeffrey A. Nystuen, Chair \\ Univ. of Washington, Applied Physics Lab., 1013 NE 40th St., Seattle, WA 98105-6698 \\ Chair's Introduction-1:35 \\ Invited Papers
}

1:40

1pAO1. The origin of storm wave noise and its application to passive acoustic remote sensing of bubbles. Grant Deane and Helen Czerski (Scripps Inst. of Oceanogr., Code 0238, UCSD, La Jolla, CA 92093-0238)

Breaking waves in storms create large numbers of bubbles at the ocean surface. Direct measurement of the bubbles is difficult, and there are few data on the numbers, sizes, and creation rates of the large, transient bubbles formed in whitecaps. These bubbles could be measured using passive acoustic remote sensing if the excitation mechanism of sound production from newly created bubbles was understood. Using bubbles released from a nozzle as a model system, we have determined that sound production is driven by the collapse of an air neck formed immediately after bubble pinch-off. Laboratory experiments and model calculations of the mechanism will be presented. [Work supported by ONR and NSF.]

\section{2:00}

1pAO2. Acoustic surface-interaction phenomena during storms: A semi-empirical approach. Roger Gauss and Joseph Fialkowski (Naval Res. Lab., Code 7144, Washington, DC 20375-5350)

When storms arise at sea, winds transfer energy to the water surface, raising waves that grow higher and longer, and generating subsurface bubbles via breaking waves. This increased complexity of the undersea air-sea boundary-interaction zone makes it important to be able to isolate and characterize the contributions of the rough air-sea interface, bubbles, and fish on an impinging acoustic field to allow realistic assessments of their impact on sonar system performance. Active transmissions can be used to remotely sense this environment and help decipher the interaction process. In this paper, a coupled approach is presented that provides self-consistent oceanographic and acoustic models. Through a combination of oceanographic and acoustic measurements and modeling, semi-empirical oceanographic models of key phenomena (wave age, air-void fraction) are first developed that depend on readily-observable environmental parameters (wind speed and significant wave height). In turn, these models are used to develop broadband semi-empirical acoustic scattering and loss models that environmentally depend on these same parameters. The paper concludes with illustrations of how strong this dependence is during storm conditions, as well as the extent to which other scattering phenomena (fish) can be masked. [Work supported by ONR.]

\section{Contributed Paper}

2:20

1pAO3. Multibeam sonar observations of near-surface bubbles during a storm. Thomas C. Weber (Ctr. for Coastal and Ocean Mapping, Univ. of New Hampshire, 24 Colovos Rd., Durham, NH 03284)

In October 2006, a reson SeaBat 7125 multibeam sonar $(400 \mathrm{kHz})$ mounted on the drop keel of the R/V Hugh Sharp was used to collect acoustic backscatter from bubbles generated by breaking wind-waves in the Gulf of Maine. The multibeam sonar has a very high spatial resolution, with $2561.0 \times 0.5 \mathrm{deg}$ beams. The data of interest here were collected as the ship returned to port because of bad weather (sustained $15 \mathrm{~m} / \mathrm{s}$ wind speed), traveling through the plumes of breaking waves. Data were collected at a ping rate that varied between $5-10$ pings/s. These observations show frequent breaking wave bubble plumes extending up to $12 \mathrm{~m}$ deep. The data also show that preferential concentrations of bubbles (clusters) were often present. Correlation functions (e.g., the pair correlation) describing the bubble clustering have been extracted from this data, and their affect on acoustic propagation characteristics (sound speed, attenuation) will be presented. [Work supported by ONR Ocean Acoustics.] 


\section{Invited Papers}

2:35

1pAO4. Acoustic monitoring of severe weather in the northeast Pacific Ocean. Jeffrey A. Nystuen (Appl. Phys. Lab., Univ. of Washington, Seattle, WA 98105, nystuen@apl.washington.edu) and Svein Vagle (Fisheries and Oceans Canada, Sidney, BC, Canada V8L 4B2)

Wind and rainfall are the principal physical processes responsible for the production of high frequency $(1-50 \mathrm{kHz})$ ambient sound in the ocean. The primary source of the sound is the resonant ringing of individual bubbles created during wave breaking and raindrop splashes. Larger bubbles (?‘ $300 \mu$ diam) quickly return to the surface, while smaller bubbles can be mixed downward many meters. During severe weather, a layer of smaller ambient bubbles forms and effectively absorbs higher frequency $(>10 \mathrm{kHz})$ sound. These processes are revealed in a two-year record of ambient sound recorded from a sub-surface mooring at $50 \mathrm{~N}, 145 \mathrm{~W}$ in the northeast Pacific Ocean as part of the Canadian SOLAS program. The passive acoustic signal of wind, rain, and ambient bubble clouds are compared to the sub-surface mooring data including data from an upward looking $200 \mathrm{kHz}$ active sonar and a $300 \mathrm{kHz}$ ADCP. The acoustic signatures of light, moderate and heavy rainfall are superimposed on the signature of high wind, demonstrating rainfall detection even in the presence of high wind. [Work supported by ONR, Fisheries and Oceans Canada, and the Canadian CFCAS \& NSERC.]

\section{2:55}

1pA05. Quantifying the destructive power of hurricanes with naturally occurring undersea sound. Nicholas C. Makris and Joshua D. Wilson (Massachusetts Inst. of Technol., Cambridge, MA 02139, makris@mit.edu)

A passive ocean-acoustic method for quantifying the destructive power of a hurricane with accuracy similar to that of in situ aircraft measurements is presented. The method has been demonstrated by comparing underwater acoustic data with aircraft wind speed data. Both the acoustic and wind speed data were obtained in 1999, when hurricane Gert passed over an autonomous underwater hydrophone in the North Atlantic. The implications of this relatively safe and inexpensive ocean acoustic method for hurricane classification and disaster planning are discussed. A new experimental initiative between Mexico and the United States on ocean acoustic hurricane quantification will be described. [This work is supported by the Office of Naval Research and Seagrant.]

\section{Contributed Papers}

3:15

1pAO6. Comparison of wind driven ambient noise in shallow and deep-water ocean environments. David Knobles, Steven E. Cho, Sumedh M. Joshi (Appl. Res. Labs., Univ. of Texas, P.O. Box 8029, Austin, TX 78713), Roy Gaul (BlueSea Cooporation, Houston, TX 77014), and Brian J. Sperry (Sci. Application Intl., Inc., McLean, VA)

Measured sound pressure time series in shallow and deep-water ocean environments are used to investigate the character of wind-generated noise over a range of wind speed of 2-40 knots and a frequency band of 103,000 Hz. In 2006, acoustic data were collected during tropical storm Ernesto off the New Jersey continental shelf in shallow $(70 \mathrm{~m})$ water. Ambient noise from these data is compared to deep-water measurements at several locations in the Northwest and the Northeast Pacific. The results also are compared to previously reported measurements by [Kuperman and Ferla (1985)], [Wenz (1962)], and others. The analysis includes a technique for separating background shipping noise from the windinduced component of ambient noise. The system electronic noise floor and other extraneous sources of noise are evaluated to assure that the ambient noise measurements are not corrupted. Physical mechanisms examined include a wind-saturation effect that limits the ability to acoustically monitor distant storms and the effect of shallow water propagation on the character of wind noise. The identification of the distant shipping part of the spectrum allows for inferences to be made on the low frequency part of the ambient noise spectrum. [Work supported by ONR.]
3:30

1pA07. High resolution acoustic characterization and change detection to evaluate hurricane effects on a coastal bay bottom. Angelina M. Freeman, Harry H. Roberts (Coastal Studies Inst., Dept. of Oceanic and Coastal Sci., Louisiana State Univ., Baton Rouge, LA 70803, afreem8@1su.edu), and Patrick D. Banks (Louisiana Dept. of Wildlife and Fisheries, Baton Rouge, LA 70898)

Using high-resolution acoustics (sidescan sonar, chirp sonar subbottom, and echo sounder profile data) the shallow surface and subsurface of Sister Lake (Terrebonne Basin) of coastal Louisiana's deltaic plain was imaged pre- and post-hurricanes Katrina and Rita. The pre-hurricanes survey in May 2005 imaged the entire lake bottom. To assess the effects of hurricanes Katrina and Rita, a second survey in October 2005 included acoustic resurveying of corridors of data covering all major sectors of the lake. Regions of sidescan reflectance differences between pre- and poststorm periods, corroborated with regions of onlapping sediment or zones of scour in the chirp sonar subbottom data, show areas both of sedimentation and erosion between surveys. Ground truth box cores were collected to calibrate acoustic data. Preliminary image processing results of the georeferenced pre- and post-storms sidescan datasets indicate $60 \%$ of the area experienced sedimentation and $40 \%$ was scoured between surveys. Amplitude reflectivity of chirp horizons were mapped, and incorporated with sidescan amplitude and texture attributes in change detection analysis. Standardized digital processing, supervised classification, and change detection techniques are developed to characterize the bay bottom and shallow subsurface and to assess and quantify storm effects. [Work supported by NOAA NA03NMF4520316.] 


\title{
Session 1pPA
}

\section{Physical Acoustics and Acoustical Oceanography: Acoustic Applications for Hurricane and Storm Preparedness}

\author{
Preston S. Wilson, Chair \\ Univ. of Texas at Austin, Dept. of Mechanical Engineering, 1 University Station, Austin TX 78712-0292
}

Invited Papers

4:00

1pPA1. Monitoring hurricanes using infrasound. Carrick L. Talmadge, Claus Hetzer, Kenneth E. Gilbert, and Roger Waxler (Univ. of Mississippi, Natl. Ctr. for Physical Acoust., Oxford, MS 38677, clt@olemiss.edu)

The University of Mississippi is leading a multi-institutional collaboration to monitor hurricane activity using infrasound, in particular the microbarom signal produced by any strong cyclonic storm. Monitoring technologies currently in use do not permit accurate long-term predictions of hurricane intensity, nor in general even for the rapid warning of sudden intensification of the storm strength. The current status of the hurricane acoustic monitoring system, including advances in sensor technology at the NCPA, will be reviewed. The potential for microbaroms to be used as an indicator of storm strength and as a metric to aid in predicting future changes in storm strength will be discussed.

4:20

1pPA2. Underwater surveys after the storm. Peter J Alleman (C \& C Technologies, Inc., 730 E. Kaliste Saloom Rd., Lafayette, LA 70508)

Following hurricanes Katrina and Rita, many types of acoustic systems were used to assess the effects of the storms. Swath multibeam sonars were used to survey erosion in ports, channels, and estuaries. Sidescan and scanning imaging sonars were used to locate debris, obstructions, missing oil platforms and ships, and dislocated pipelines. Acoustic positioning systems and acoustic modems were used to support the sonar imaging systems. A wide array of acoustic systems were used in the overall effort. Samples of data from several types of sonar systems will be presented along with discussions of the challenges of collecting data in a real-world environment.

\section{Contributed Papers}

\section{4:40}

1pPA3. Infrasound measurements during hurricane Katrina. Ronald Wagstaff, Eric Goggans, Heath Rice, and Carrick Talmadge (NCPA, Univ. of Mississippi, 1 Coliseum Dr., University, MS 38677, rwagstaf@olemiss.edu)

Infrasound is used to detect both natural and man-made events. Microbarometric measurement stations have been placed worldwide in order to detect infrasonic signals in the atmosphere. Such signals range in frequency from less than 0.01 to $10 \mathrm{~Hz}$. Infrasound is produced by many natural phenomena including violent storms, gravity waves, the Aurora Borealis, and even some animals. Because infrasound signals have such low frequencies, they can travel great distances in the atmosphere, while maintaining good temporal coherence. This makes the atmosphere an excellent medium for infrasound signal propagation and corresponding detection of large storms, such as hurricanes and tornados, which generate strong infrasonic signals. This paper will discuss hurricane Katrina's infrasound spectral results obtained from data measured by piezo-ceramic infrasound sensors in Oxford, MS, about 400 miles to the north of New Orleans. Spectral and temporal coherence results will be presented and discussed. [Work supported by ARDEC.]
$4: 55$

1pPA4. Generation of microbaroms by hurricanes. Claus Hetzer, Carrick L. Talmadge, Kenneth E. Gilbert, and Roger Waxler (Natl. Ctr. for Physical Acoust., Univ. of Mississippi, Coliseum Dr., University, MS 38677)

Under quiet conditions, a background peak at approximately $0.2 \mathrm{~Hz}$ is observed worldwide on infrasound stations. The peak is understood to be the "microbarom peak" associated with ocean waves. The intensity of microbaroms increases dramatically on inland infrasound stations when a hurricane is offshore and within a few thousand $\mathrm{km}$. Hurricanes, thus, are unique phenomena for investigating microbarom generation by ocean waves. We review recent microbarom data and examine existing theories for the generation of microbaroms. In particular, we propose two possible models for the generation of microbaroms by hurricanes. In the first model, the microbaroms are generated near the eye of the hurricane, and in the second model, by counter propagating ocean wave fields created by the hurricane and relatively far from the eye. Bearing data from infrasound stations are presented that support the second model. [Supported by the U.S. Army Space and Missile Defense Command.] 


\title{
Session 1pUWa
}

\section{Underwater Acoustics: Geoacoustic Inversion}

\author{
David P. Knobles, Chair \\ Environmental Sciences Dept., Applied Research Labs., Univ. of Texas at Austin, Austin, TX 78713 \\ Chair's Introduction-1:00
}

Contributed Papers

1:05

1pUWa1. Analyses of broadband and narrowband propagation measurements in Shallow Water 2006 (SW06). David P. Knobles, Robert A. Koch, Preston S. Wilson (Appl. Res. Labs., Univ. of Texas, P.O. Box 8029, Austin, TX 78713), Timothy F. Duda, and Arthur E. Newhall (Woods Hole Oceanogr. Inst., Woods Hole, MA 02543)

Acoustic measurements were made during the Shallow Water 2006 Experiment near the New Jersey continental shelf in 70-80 m of water. For a portion of the experiment, several L-arrays and a variety of broadband and narrowband sources were deployed for the purpose of investigating the nature of the dispersion of the acoustic propagation in the seabed. A geo-acoustic inversion methodology is used to infer the dispersion from the acoustic measurements in the water column. The waveguide possesses significant inhomogenities in range, azimuth, and time due to bathymetry, sound speed profile fluctuations resulting from internal waves, and variability in seabed properties. Physical oceanography measurements are used in part to obtain the best estimate for a water column description for the analyses of specific acoustic measurements. Various inversion methods along with different propagation models, such as two-way coupled modes and finite element parabolic equation algorithms, are used to obtain an accurate mean description and uncertainty estimates for the properties that control dispersion of the seabed. [Work supported by ONR.]

\section{$1: 20$}

1pUWa2. Is higher resolution bathymetry always better for undersea sound propagation modeling? Juan Arvelo (Appl. Phys. Lab., Johns Hopkins Univ., 11100 Johns Hopkins Rd., Laurel, MD 20723)

Bathymetry is one of the most important environmental parameters affecting undersea sound transmission in shallow waters. There has always been a preference towards bathymetry with the highest resolution and accuracy when conducting sound propagation modeling. However, extreme care must be exercised in the selection of the correct bathymetric resolution. It will be demonstrated that the correct resolution depends on the source of the geoacoustic model used. Higher resolution bathymetrics, such as multibeam surveys, introduce steeper slopes that yield larger bottom losses. If a lower resolution bathymetry was implemented to conduct geoacoustic inversion, then the same bathymetric resolution must be used to conduct subsequent sound transmission modeling. In addition, when geoacoustic inversion results are reported, the bathymetric resolution used should also be reported.

\section{$1: 35$}

1pUWa3. An autonomous underwater vehicle as sound source for geoacoustic inversion. A. Vincent van Leijen (NLDA, P.O. Box 10000, 1780 CA, Den Helder, The Netherlands)

During the MREA07 trials an autonomous underwater vehicle (AUV) was deployed to run a bathymetrical survey in a shallow water environment off the coast of Italy. Apart from much coastal and recreational activity in the area, self-noise of the REMUS AUV was continuously observed on a sparse vertical receiver array that was deployed from a small boat at anchor. As the vehicle ran its survey with a speed of $5 \mathrm{kts}$ and at a depth of $30 \mathrm{~m}$, the propulsion system radiated harmonic related tones of low frequencies that were received in strong interaction with the sea bottom. Three selected tones were then exploited for the geoacoustic inversion of a local range-independent environmental model. The inverted parameters describe a soft sediment layer on top of a hard sub bottom; the obtained model with specified uncertainty is shown to match seismic and bathymetric data of the area.

\section{1:50}

1pUWa4. Inversion of sediment properties from observations of scattering of buried cylinders using subcritical ensonification. William Sanders, Dale Bibee (Naval Res. Lab., Sea Fl. Sci. Branch, Stennis Space Ctr., MS 39529), and David Calvo (US Naval Res. Lab., Washington, DC 20375)

It is well known that objects buried near the water-sediment interface may be ensonified beyond the critical angle. Although the evanescent portion of surface waves decays rapidly with depth, objects near the surface may reradiate significant amounts of energy, especially at lower frequencies. Moreover, surface roughness may serve as a mechanism for enhancing the penetration of energy at higher angles. This study addresses the viability of inferring and then utilizing geo-acoustic properties from such scattering observations. A synthetic data set is generated for testing inversion using genetic algorithms (using the SAGA toolkit developed by Peter Gerstoft) and by use of a new model for predicting scattering from buried cylinders, target response using matched asymptotics. Distributions of posterior errors in the inverted parameters are presented. [This work was supported in part by the Naval Research Laboratory under program element PE62782N.]

2:05

1pUWa5. Accuracy of passive matched-field, thick-sediment inversions in range-dependent environments. Donald Del Balzo and Lisa Pflug (Planning Systems Inc., 40201 Hwy 190 East, Slidell, LA 70461)

Accuracy of shallow-water acoustic predictions is severely limited by uncertainty in sediment property characteristics. Recent research, using inverse methods to invert signals from controlled active sources, has been conducted to estimate seabed properties. However, some applications prefer a more covert approach. Our work is focused exclusively on passive, inversion techniques using signals from surface ships-of-opportunity. This study addresses the accuracy of low-frequency $(\sim 100 \mathrm{~Hz})$ matched-field correlations using broadband signals from surface ships with unknown source levels at unknown ranges. A range-staggered technique is employed to create a range-dependent sediment description, with ever increasing confidence as additional observations are made. Matched-field techniques are applied in a simulated shallow-water environment with a single vertical line array and high signal-to-noise ratios. The simulations indicate 
significant potential for accurate estimates of thick-sediment characterizations of grain size and layer thickness out to ranges of tens of water depths in shallow water, despite moderate mismatch conditions in the environmental model. [Work sponsored by SPAWAR.]

\section{$2: 20$}

1pUWa6. Bottom and sub-bottom roughness effects on coherence and geo-acoustic inversions. Kevin D. Heaney (OASIS Inc., 11006 Clara Barton Dr., Fairfax Station, VA 22039)

In this discussion, the effect of bottom and sub-bottom roughness on acoustic propagation in shallow water is examined. Generally in underwater acoustics, the acoustic medium is quantized as a stratified medium, with dominant changes occuring in depth and the range-dependent envi- ronmental variability (apart from bathymetry) is neglected. For a few environments presented in this paper, this approximation is quite poor. Scattering from bottom and sub-bottom roughness can lead to loss of signal and is often treated as an anomolous attenuation. Energy scattered from a rough hard bottom or basement is not removed from the water column but instead propagates as incoherent energy reducing the measured coherence and leading. A numerical model that incorporates random bathymetric and sub-basement roughness into the parabolic equation is presented. Comparisons of the effects of roughness will be made for an experiment over Basalt (BASSEX2004) and limestone (CALOPS 2007), both of which are thought to be hard, rough basements, covered with sand. Comparisons of measured and predicted coherence, geo-acoustic inversion results, and eigenspectra will be presented.

TUESDAY AFTERNOON, 27 NOVEMBER 2007

GRAND BALLROOM D, 3:00 TO 4:20 P.M.

\title{
Session 1pUWb
}

\section{Underwater Acoustics: Rough Surface Interface Scattering}

\author{
Guy V. Norton, Chair \\ Acoustics Div., Naval Research Laboratory, Stennis Space Center, MS 39529-5004
}

Chair's Introduction-3:00

\section{Contributed Papers}

3:05

1pUWb1. Acoustic scattering and propagation through spatially complex dispersive media via the direct numerical solution of the wave equation. Guy V. Norton (Naval Res. Lab., Stennis Space Ctr., MS 39529, guy.norton@nrlssc.navy.mil)

Analytical approaches for modeling propagation and scattering in inhomogeneous media are difficult, and often require extremely simplifying approximations in order to achieve a solution. To avoid such approximations, the direct numerical solution of the wave equation via the method of finite differences offers the most direct tool, which takes into account diffraction and refraction. It also allows for detailed modeling of spatially complex media. In all cases, the correct inclusion of the dispersive properties of the medium can make the difference in the interpretation of the results. However, the inclusion of dispersion directly in the time domain proved until recently to be an elusive problem. In order to model the transient signal, a convolution operator that takes into account the dispersive characteristics of the medium is introduced to the linear wave equation. In this work, numerical modeling of scattering from a variety of different dispersive media with physical properties associated with real world material is presented. Where possible, the numerical solutions are compared with a known solution. It is shown that in those cases, the use of the convolutional propagation operator leads to the correct solution for the scattered field. [Work supported by ONR/NRL.]

\section{$3: 20$}

1pUWb2. A geometric method for calculating the Doppler spreading of surface scattered signals. James Preisig (Woods Hole Oceanograph. Inst., Woods Hole, MA 02543), Grant Deane (Scripps Inst. of Oceanogr., La Jolla, CA 92093), and Chris Tindle (Univ. of Auckland, Auckland, New Zealand)

Recent work has shown that the characteristics of surface scattered high frequency signals can significantly impact the performance of underwater acoustic communications systems in shallow water environments. A combination of high signal intensities due to the focusing of the signals by the curved surface waves and high Doppler spreads due to the motion of the surface waves make it difficult to accurately track the time varying channel impulse response as required by most high rate communications systems. However, algorithms that can model and accommodate the Doppler spreading have been shown to maintain acceptable performance in these environments. The ability to predict the expected Doppler spreads of the surface scattered signals as a function of source/receiver geometry and surface wave characteristics can improve the performance of some of these algorithms. A method for calculating the Doppler spreading of surface scattered signals as a function of the source/receiver geometry and surface wave slope, curvature, and velocity is presented. Comparisons between theoretical predictions and observed Doppler spreads in collected acoustic data are presented.

\section{3:35}

1pUWb3. Low frequency enhancement of water-to-air sound transmission. B. Edward McDonald and David Calvo (Naval Res. Lab., Washington, DC 20375)

Low-frequency acoustic radiation into the air from an underwater point source is investigated using plane wave expansion of the source spectrum and Rayleigh reflection/transmission coefficients in order to clarify a recent claim of "anomalous transparency" of the water-air interface at low acoustic frequencies [Phys. Rev. Lett. 97, 164301, 2006]. Carrying the analysis to first order in the air/water density ratio, it is found that as the depth-wave number product $\mathrm{kD}$ becomes small, radiation into the water is quenched by the source's acoustic image, while the power radiated into air increases to about one percent of the power that would be radiated into unbounded water. For $\mathrm{kD}$ less than about 0.093 , a majority of the one percent surviving total energy is transmitted to the air. In our view it seems an exaggeration to refer to this as "anomalous transparency." [Work supported by the Office of Naval Research.] 
1pUWb4. Resolving travel-time variability of the coupled oceanographic-tomographic analysis and prediction system (COTAPS). Irena Lucifredi and Peter Stein (Sci. Solutions Inc., 99 Perimeter Rd., Nashua, NH 03063)

It has been suggested that the tomographic approach that feeds the 8-11 kHz ocean acoustic measurements into an ocean model using data from bottom-mounted hydrophones, suffers from a significant travel-time variability due to the surface waves. In this work, the influence of the ocean surface motion on the acoustic travel times has been studied using both the experimental data collected at the Pacific missile range facility (PMRF) off Kauai, and an appropriate acoustic model. The ray-based acoustic model developed is an adaptation of the range-dependent version of Bellhop with the modified top surface characterizing ocean surface waves. The model-generated time series suggests a direct relationship between the phase of the top surface wave and the time-of arrival, which is further confirmed by the experimental data. Furthermore, an approach is developed that automatically decouples the effect of the top surface waves from the environment-related phenomena, making it possible to assign the travel time variations produced by the physical phenomena independently of the surface geometry, in turn eliminating the surface related error. [Work supported by the ONR.]
1pUWb5. Effect of rough surface on received signals. Jeremy $M$. Joseph, Richard Lee Culver, Jeffrey A. Ballard, and Colin W. Jemmott (Grad. Program in Acoust., The Penn State Univ., PO Box 30, State College, PA 16804,jmj269@psu.edu)

The overall goal of our research is to incorporate knowledge of variability and uncertainty in received passive sonar signals into the signal processing. As part of this goal, we need to better understand the sources of this variability. It is well known that wave motion on the ocean surface can introduce significant effects on undersea acoustic signals. This talk focuses on predicting the impact of rough ocean surfaces on the variability of the received signal. An acoustics propagation code, range-dependent acoustic model (RAM) rough surface fine coarse (RRSFC), is utilized to simulate environments with flat and rough ocean surfaces. To explore the effects of random rough surfaces, a Monte Carlo method is applied to yield environment realizations with varying rough surfaces. From these simulations, probability density functions are generated and are compared with amplitude figures from environments with a flat surface in order to distinguish any overall effect on a received signal. [Work supported by ONR Undersea Signal Processing Code 321US.]

Note: Payment of additional registration fee required to attend tutorial.

TUESDAY EVENING, 27 NOVEMBER 2007

GRAND BALLROOM E, 7:00 TO 9:05 P.M.

Session 1eID

Interdisciplinary: Tutorial Lecture on Weather and Acoustics

\author{
James P. Chambers, Chair \\ National Center for Physical Acoustics, Univ. of Mississippi, 1 Coliseum Dr., University, MS 38677
}

Chair's Introduction-7:00

Invited Paper

7:05

1eID1. Tutorial lecture on weather and acoustics. Alfred J. Bedard (NOAA Environ. Technol. Lab., 325 Broadway, Boulder, CO 80303)

The relationships between sound and weather can be fascinating, frightening, useful, and at times mystifying. This tutorial lecture explores the range of intersections between weather and acoustics. Weather can affect acoustic environments causing noise increases, noise reductions, and sound focusing. One aspect of this tutorial reviews results from propagation modeling, indicating that under some conditions, the atmosphere can produce vertical wave guides. Conversely, sound can be used to actively interrogate the atmosphere and provide information valuable for weather prediction and warning. Probing capabilities reviewed, with examples, show that wind profiles, temperature profiles, wind shears, gravity waves, and inversions can be defined acoustically. There are also possibilities for monitoring other difficult-to-observe parameters such as humidity profiles. In addition, weather processes can generate sound, detectable at long ranges using lower frequencies. Specifically, observing networks have observed infrasound from a growing number of meteorological events (e.g., severe weather, tornadoes, funnels aloft, atmospheric turbulence, hurricanes, and avalanches). Efforts to develop an infrasonic tornado detection system are described in some detail. Results indicate promise to help improve tornado detection and warning lead times, while reducing false alarms. Clear opportunities exist for infrasonic systems to provide operational weather data. 